\title{
Management of novel oral anticoagulants in the perioperative setting
}

\begin{abstract}
The perioperative management of Novel Oral Anticoagulants (NOACs) is an area of continuing research and there is yet to be a concrete protocol for any of the agents in place. These agents being dabigatran, rivaroxaban and apixaban. The aim of this review is to summarise the literature to date and give guidance as to what is currently recommended. Of course, findings differed depending on the agent of interest but all related to bleeding risk and creatinine clearance. Cessation of dabigatran varied from 24-96 hours and 24144 hours for standard and high bleeding risks, respectively. Each group further divided by creatinine clearance. Re-introduction is also dependent on bleeding risk with a lower dose on the evening of a low, or otherwise known as standard, risk procedure and a normal dose the following day. High risk is guided by haemostasis. Much less variation was seen for rivaroxaban and apixaban. A preoperative cessation of 24 hours for standard bleeding risk and 48 hours for high was advised for both regardless of creatinine clearance. This is with the exception of a creatinine clearance of $\leq 30 \mathrm{ml} . \mathrm{min}-1$ in a standard risk procedure, for which a time frame of 36 hours was suggested. Re-introduction for both is once again determined by haemostasis. It should be noted these times pertain only to elective surgery. The management of NOACs in emergency surgery remains largely unknown. Though not set in stone this is a starting point and can give us direction going forward as the popularity of NOACs grows.
\end{abstract}

Keywords: NOAC, dabigatran, rivaroxaban, apixaban, perioperative
Volume 8 Issue 4 - 2017

\author{
Elyse Bond,' Glenn Hurtado,' Shanthi \\ Kulasinghe,' Thomas Melhuish, ${ }^{2,3}$ Ruan Vlok,, ${ }^{2,4}$ \\ Leigh White ${ }^{1,5}$ \\ 'Caboolture Hospital, Australia \\ ${ }^{2}$ Wagga Wagga Rural Referral Hospital,Australia \\ ${ }^{3}$ University of New South Wales, Australia \\ ${ }^{4}$ University of Notre Dame, Australia \\ ${ }^{5}$ University of Wollongong, Australia
}

Correspondence: Leigh White, University of New South
Wales, University of Wollongong, Australia,

Email lw844@uowmail.edu.au

Received: April 20, 2017 | Published: September 12, 2017

\section{Introduction}

For decades warfarin has been the oral anticoagulant of choice. However, in recent years there has been a significant shift towards the use of NOACs. Unlike most other medications, such as warfarin and anti-hyperglycaemic agents, ${ }^{2}$ the protocol for perioperative cessation of NOACs remains unclear. The aim of this review is to summarise a growing body of evidence surrounding the perioperative management of NOACs; dabigatran (Pradaxa), rivaroxaban (Xarelto) and apixaban (Eliquis). NOACs hold many advantages over warfarin in the management of procoagulant disorders, such as their rapid onset with no bridging required, short half-life allowing timely cessation and not requiring monitoring blood tests. ${ }^{3-5}$ The advantage of not requiring monitoring blood tests is, however, a disadvantage in the perioperative setting, as coagulation studies are therefore a poor predictor of agent reversal. ${ }^{6}$ What's more, reversal is difficult. The cessation of these agents is currently based on their relatively predictable pharmacokinetics, which is strongly related to renal function. ${ }^{7,8}$

\section{Dabigatran}

Dabigatran is a direct thrombin inhibitor (factor IIa). ${ }^{3}$ Two large studies have investigated the management of dabigatran during the perioperative period, one a subsection of the RE-LY study ${ }^{3}$ and the other by Schulman et al. ${ }^{9}$ A subgroup of the RE-LY study ( $\left.n=4591\right)$ had either dabigatran or warfarin for non-valvular AF ceased prior to a range of procedures of both low and high bleeding risk. The incidence of major bleeding with warfarin, dabigatran $110 \mathrm{mg}$ and dabigatran $150 \mathrm{mg}$ was $4.6 \%, 3.8 \%$ and $5.1 \%$, respectively. The 30 -day incidence of embolism following the discontinuation period was $0.5 \%$ in each group.

The study by Schulman and colleagues was a prospective study of 541 patients, which included standard and high risk bleeding procedures. ${ }^{9}$ This study showed lower rates of major bleeding (1.8\%) than the RE-LY study but similar low rates of thromboembolism of $0.2 \%$. The protocol for discontinuation in these studies was based on the risk of procedural bleeding and the patients creatinine clearance $(\mathrm{CrCl})$ (Table 1). ${ }^{3,7,8}$ The cessation of dabigatran varied between one and six days prior to surgery. The decision to reintroduce dabigatran requires careful consideration as it has a rapid onset of action with peak effect reached within two to three hours. In these studies, dabigatran was commonly restarted at a lower dose (e.g. $75 \mathrm{mg}$ ) on the evening of a low risk procedure and their normal dose resumed the day following the procedure. ${ }^{3}$ Patients who underwent a high bleeding risk procedure had the re-introduction of dabigatran guided by surgical haemostasis. ${ }^{3}$ Most patients resumed their normal dose by two to three days post operation.

\section{Rivaroxaban}

Rivaroxaban is one of the direct factor Xa inhibitors, ${ }^{4}$ the other being apixaban. ${ }^{5}$ Currently, there is only one study investigating the effect of perioperative cessation of rivaroxaban, which is a subgroup of the ROCKET-AF study. ${ }^{4}$ This study used greater periods of cessation than the dabigatran studies, with rivaroxaban ceased for greater than three days prior to surgery, despite possessing a half-life slightly shorter than dabigatran (Table 1). ${ }^{8}$ This is because rivaroxaban has a significantly higher protein binding than dabigatran. ${ }^{8}$ The ROCKETAF study showed no significant difference in major haemorrhage or thromboembolic events. Since this study several regiments for discontinuation have been proposed (Table 1). ${ }^{4,7,8}$ These regiments are significantly shorter than those used by the ROCKET-AF study and are, therefore, not supported by published data on the incidence of haemorrhage and thromboembolism. One unique characteristic of rivaroxaban worth consideration during the perioperative period is its interaction with dual inhibitors of P-glycoprotein and CYP-3A4 (e.g. antiviral and antifungal agents). ${ }^{7,8}$ The re-introduction of rivaroxaban 
is guided by haemostasis given its rapid onset of action with peak levels achieved 2-4 hours post ingestion. ${ }^{7,8}$

Interestingly, there is new data arising for rivaroxaban which may lead it and the other NOACs to be indicated for coronary artery disease. ${ }^{11}$ Given that coronary artery disease has many classifications, ${ }^{12-14}$ no ceasation protocols can be recommended until more concrete indications for the use of NOACs have been determined.

\section{Apixaban}

Currently, only one study is available investigating perioperative cessation of apixaban. ${ }^{5}$ The Aristotle trial $(n=9260)$ primarily assessed low risk procedures in which cessation occurred in $60 \%$ of these. A cessation of 2-5 days pre-operation was used. ${ }^{7,8}$ This study compared the outcomes of patients on either apixaban or warfarin irrespective of whether discontinuation had occurred. The period in which patients discontinued apixaban was different to recommendations made based on pharmacokinetic studies, ${ }^{7,8}$ which ranged from 24 to 48 hours (Table 1). Similar to rivaroxaban, re-introduction is guided by haemostasis given its rapid onset of action with peak levels achieved 1-4 hours post ingestion. ${ }^{7,8}$

\section{Emergency surgery}

One of the main concerns related to the prescribing of NOACs is their relative unpredictability if emergency surgery is required. Currently there are several key unknowns to NOACs that make other anticoagulants, such as warfarin, more favourable to manage in an emergency situation. ${ }^{6}$ These include the uncertainty of safe operative NOAC concentrations, and the inaccuracy of coagulation studies to determine the anticoagulant effect. ${ }^{6}$

Currently, there is no consensus on how NOACs should be reversed in the case of emergency surgery or catastrophic bleeding. Prothrombin complexes have some evidence supporting its use,$^{10}$ however its use comes with a significant risk of thromboembolic events. Similarly, tranexamic acid may also reduce the risk of bleeding however there is no clinical data to support its use. Dabigatran is unique, as it is the only NOAC that can be successfully removed via haemodialysis if timing permits. ${ }^{14}$ This is due to its relatively minimal protein binding of $35 \%{ }^{8}$ Dabigatran is also unique as it is the only NOAC that has a commercially available reversal agent, Idarucizumab. Only one small clinical study has been performed and the results are promising. ${ }^{15}$ This study of 36 patients, using $5 \mathrm{~g}$ of idarucizumab, showed successful preoperative reversal where only three patients exhibited mild to moderately abnormal haemostasis. Only one thromboembolic event occurred within three days of reversal. Rivaroxaban and apixaban do not currently have a commercially available reversal agent. However, preliminary studies have been performed on an agent named andexanet. These have demonstrated a rapid reduction in plasma concentration of rivaroxaban and apixaban following administration. ${ }^{16}$ But until these reversal agents become available it is best to, cease the NOAC, postpone the surgery where possible, and then consider haemostatic factors and/or dialysis, in that order. ${ }^{17}$

Table I Perioperative cessation of NOACs according to bleeding risk and creatinine clearance

\begin{tabular}{|c|c|c|c|}
\hline Creatinine Clearance $\left(\mathrm{ml} . \mathrm{min}^{-1}\right)$ & Estimated Half-Life, hours (range) & Standard Bleeding Risk & High Bleeding Risk \\
\hline \multicolumn{4}{|l|}{ Dabigatran } \\
\hline$>80$ & $13(||-22)$ & $24 \mathrm{hrs}$ & $48 \mathrm{hrs}$ \\
\hline $51-80$ & $15(12-34)$ & 24 hrs (2 doses) & $48-72 \mathrm{hrs}$ \\
\hline $31-50$ & $18(13-23)$ & $48 \mathrm{hrs}$ & $96 \mathrm{hrs}$ \\
\hline$\leq 30$ & $27(22-35)$ & $48-96 \mathrm{hrs}$ & I20-|44 hrs \\
\hline \multicolumn{4}{|l|}{ Rivaroxaban } \\
\hline$>80$ & I3-May & $\geq 24$ & $\geq 48$ \\
\hline $51-80$ & 8.7 & $\geq 24$ & $\geq 48$ \\
\hline $31-50$ & 9 & $\geq 24$ & $\geq 48$ \\
\hline$\leq 30$ & 9.5 & $\geq 36$ & $\geq 48$ \\
\hline \multicolumn{4}{|l|}{ Apixaban } \\
\hline$>80$ & 12 & $\geq 24$ & $\geq 48$ \\
\hline $51-80$ & 14.6 & $\geq 24$ & $\geq 48$ \\
\hline $31-50$ & 17.6 & $\geq 24$ & $\geq 48$ \\
\hline$\leq 30$ & 17.3 & $\geq 36$ & $\geq 48$ \\
\hline
\end{tabular}

\section{Conclusion}

The management of the aforementioned NOACs in the perioperative setting varies as a result of the limited evidence available. That said, by reviewing the current literature we are able to formulate an evidence-based guide as seen in Table 1. The timing of cessation for each is determined by anticipated bleeding risk of the surgery and the $\mathrm{CrCl}$ of the patient involved. Re-introduction of the agents is usually based on haemostasis post operatively. Except for dabigatran after a low or standard risk procedure in which a lower dose can be taken the evening of, and the full dose the day after, the surgery. More research is obviously needed but this can be used until a gold standard is produced.

\section{Acknowledgments}

None.

\section{Conflicts of interest}

Authors declare that there is no conflict of interest.

\section{References}

1. Keeling D, Baglin T, Tait C, et al. Guidelines on oral anticoagulation with warfarin - fourth edition. Br J Haematol. 2011;154(3):311-324.

2. White L. Peri-operative management of the surgical patient with diabetes: a guideline summary. J Clin Endocrinol Metab. 2012;6(3):97-99.

3. Healey JS, Eikelboom J, Douketis J, et al. Periprocedural bleeding and thromboembolic events with dabigatran compared with warfarin: results from the Randomized Evaluation of Long-Term Anticoagulation Therapy (RE-LY) randomized trial. Circulation. 2012;126(3):343-348.

4. Sherwood MW, Douketis JD, Patel MR, et al. Outcomes of temporary interruption of rivaroxaban compared with warfarin in patients with 
nonvalvular atrial fibrillation: results from the rivaroxaban once daily, oral, direct factor Xa inhibition compared with vitamin $\mathrm{K}$ antagonism for prevention of stroke and embolism trial in atrial fibrillation (ROCKET AF). Circulation. 2014;129(18):1850-1859.

5. Garcia D, Alexander JH, Wallentin L, et al. Management and clinical outcomes in patients treated with apixaban vs warfarin undergoing procedures. Blood. 2014;124(25):3692-3698.

6. Kitchen S, Gray E, Mackie I, et al. Measurement of non-coumarin anticoagulants and their effects on tests of Haemostasis: Guidance from the British Committee for Standards in Haematology. Br J Haematol. 2014;166(6):830-841

7. Heidbuchel H, Verhamme P, Alings M, et al. Updated European Heart Rhythm Association Practical Guide on the use of non-vitamin K antagonist anticoagulants in patients with non-valvular atrial fibrillation. Europace. 2015;17(10):1467-1507.

8. Lai A, Davidson N, Galloway S, et al. Perioperative management of patients on new oral anticoagulants. Br J Surg. 2014;101(7):742-749.

9. Schulman S, Carrier M, Lee AY, et al. Perioperative Management of Dabigatran:AProspective Cohort Study. Circulation. 2015;132:167-173.

10. Eikelboom J. Rivaroxaban plus aspirin improves outcomes in stable cardiovascular disease. ESC Congress; 2017.
11. White LD, Wall J, Melhuish TM, et al. Recognition and management of posterior myocardial infarction: a retrospective cohort study. British Journal of Cardiology. 2017;24:72-74.

12. Wall J, White L, Lee A. Novel ECG changes in acute coronary syndromes. Would improvement in the recognition of 'STEMI-equivalents' affect time until reperfusion?. Internal and Emergency Medicine. 2016;1-7.

13. Wall J, Vlok R, Melhuish T, et al. Acute myocardial infarction and STEMI-equivalent patterns. International Journal of Internal Medicine Papers. 2016;1:7-11.

14. Makris M. Prothrombin complex concentrate for non-vitamin K oral anticoagulant reversal: good enough for now? J Thromb Haemost. 2014;12(9):1425-1427.

15. Chang DN, Dager WE, Chin AI. Removal of dabigatran by hemodialysis. Am J Kidney Dis. 2013;61(3):487-489.

16. Pollack CV Jr, Reilly PA, Eikelboom J, et al. Idarucizumab for Dabigatran Reversal. N Engl J Med. 2015;373:511-520.

17. Siegal DM, Curnutte JT, Connolly SJ, et al. Andexanet Alfa for the Reversal of Factor Xa Inhibitor Activity. $N$ Engl J Med. 2015;373:2413-2424.

18. Aronis K, Hylek E. Who, when, and how to reverse non-vitamin K oral anticoagulants. J Thrombosis Thrombolysis. 2016;41(2):253-272. 